\title{
Differences in mitral annulus remodelling in acute anterior ST elevation and acute inferior ST elevation myocardial infarction
}

\author{
Corresponding author: \\ Ažuolas Sirtautas \\ Lithuanian University \\ of Health Sciences \\ Tel.: +37066700001 \\ E-mail: azuolas.sirtautas@gmail.com
}

Medical Research Journal 2016;

Volume 1, Number 3, 111-113

10.5603/MRJ.2016.0020

Copyright (C) 2016 Via Medica

ISSN 2451-2591

\begin{abstract}
Introduction. Myocardial infarction is known to account for left ventricular remodelling and mitral annular distortion. The aim of the study was to assess and compare morphometric changes of mitral annulus in patients presenting with acute anterior or inferior myocardial infarction. The objectives of our study were as follows: assessment of anteroposterior and septolateral annular dimensions of mitral annulus in patients with acute myocardial infarction; assessment of mitral annular area and annular contraction in patients with acute myocardial infarction; evaluation of parameters reflecting mitral apparatus (chordal length, chordal papillary muscle distance, etc.); and comparison of mitral annular geometry between patients with anterior and inferior myocardial infarction.

Materials and methods. Echocardiographic data of 30 patients with an anterior ST elevation myocardial infarction and data of 30 patients with an inferior ST elevation myocardial infarction on an acute stage was collected, evaluated, and compared.

Results and conclusions. The mitral annulus shows some significant changes in both post inferior myocardial infarction and post anterior myocardial infarction patients. The magnitude of movements of a post inferior myocardial infarction mitral annulus is greatly reduced when compared to mitral annulus in patients with anterior myocardial infarction. While echocardiographically measuring post-myocardial infarction mitral annulus, we decided that inferior myocardial infarction accounts for earlier mitral annular dilatation compared to patients with anterior myocardial infarction.
\end{abstract}

Key words: myocardial infarction, mitral annulus

Med Res J 2016; 1 (3): 111-113

\section{Introduction}

Nowadays, many people are suffering from cardiovascular diseases (CVD). Statistics presented in the European Heart Journal over the last two years report that CVD is also the most common cause of death among Europeans, and about 4 million Europeans die of CVD every year [1]. Myocardial infarction (MI) is a common occurrence and is known to account for left ventricular remodelling and mitral annular distortion (which can cause one major complication: mitral regurgitation). The mitral annulus (MA) is dynamic and undergoes conformational changes during the cardiac cycle to optimise diastolic left ventricular filling, maintain systolic competence, and minimise leaflet stress. However, clinical studies have shown an increase of size of the MA to $>30 \%$ when a patient has suffered from inferior
MI [2, 3]. An acute MI, particularly one that is large and transmural, can produce alterations in the topography of both the infarcted and non-infarcted regions of the ventricle. This remodelling can importantly affect the function of the ventricle and the prognosis for survival [4]. The main object affected by this is the MA.

The objectives of the study were to assess anteroposterior and septolateral annular dimension of MA in patients with acute $\mathrm{Ml}$ and to assess mitral annular area and annular contraction of these same patients. Also, the parameters that reflect the anatomical structure of mitral apparatus (chordal length, chordal papillary muscle distance, etc.) were evaluated. The main aim of this study was to compare various changes of mitral apparatus occurring in patients, who have been presented with acute anterior or inferior ST elevation MI. 
Table 1. Parameter difference in two-chamber ultrasound view

\begin{tabular}{lccc}
\hline Variable & $\begin{array}{c}\text { Inferior ST elevation } \\
\text { myocardial infarction }\end{array}$ & $\begin{array}{c}\text { Anterior ST elevation } \\
\text { myocardial infarction }\end{array}$ & P value \\
\hline Diastolic radius of MA & 33.956 & 31.635 & $\mathbf{0 . 0 0 0}$ \\
Systolic radius of MA & 30.599 & 30.217 & 0.339 \\
Diastolic area of MA & 9.175 & 7.901 & $\mathbf{0 . 0 0 0}$ \\
Systolic area of MA & 7.444 & 7.213 & 0.349 \\
MA contraction & 1.730 & 1.424 & 0.382 \\
Distance from AL to anterior leaflet & 29.170 & 29.080 & 0.453 \\
Distance from AL to posterior leaflet & 36.041 & 41.079 & $\mathbf{0 . 0 0 0}$ \\
Perpendicular of AL to MA & 26.255 & 27.545 & 0.202 \\
\hline
\end{tabular}

All distances are in $\mathrm{mm}$. Area is measured in $\mathrm{mm}^{2}$. Bold numbers show clinical significance; $\mathrm{MA}$ - mitral annulus; $\mathrm{AL}$ - anterolateral muscle

\section{Materials and methods}

An ultrasound was performed on patients who have been presented with acute MI. Data was collected while patients were still in an acute stage of infarction. Parameters that were used: MA was measured in systole as well as in diastole, systolic and diastolic annular area, and annular contraction. Mitral valve (MV) muscles were also evaluated using parameters: distance from anterolateral muscle to the anterior leaflet; distance from anterolateral muscle to the posterior leaflet; perpendicular of anterolateral muscle to MA. Parameters were measured using two-chamber, four-chamber, and parasternal long-axis view of the heart while using EchoPAC software.

\section{Statistical analysis}

All of the data used in this study was analysed at a separate time and had no clinical impact on patient treatment whatsoever. Statistical analysis was performed using SPSS v. 20.0 (IBM Corp, Armonk, NY, USA) software to compare parameters of mitral annulus in acute inferior and anterior STEMI. We calculated mean and Wilcoxon criteria. Statistical significance was determined at $p \leq 0.05$.

\section{Results}

A total of 60 patients were studied. While performing this study it was discovered that MA was becoming wider in patients with acute inferior Ml compared to patients with acute anterior $\mathrm{Ml}$ (for example: two-chamber view: diameter of MA in systole: $2.33 \mathrm{~mm}$ wider in inferior $\mathrm{Ml}$; diastolic area: $1.82 \mathrm{~mm}^{2}$ larger in inferior $\mathrm{Ml}$ ). The four-chamber view played a greater role in this study because when using this view it was possible to determine all of the parameters more clearly. Four-chamber view of the heart showed even more results in favour of
MA being bigger in patients with inferior MI (For example: diameter of MA measured in diastole: $6.52 \mathrm{~mm}$ wider in inferior MI). Diameter, systolic, and diastolic area of MA was also proven to be bigger in patients with inferior MI. Also, while viewing the heart in four-chamber view, it was noticed that MA was contracting stronger in patients with inferior MI. Using the two-chamber view, three out of eight of the used parameters showed clinical significance. Using the four-chamber view, five out of eight of the used parameters showed clinical significance. Using the parasternal long-axis view of the heart, four out of five used parameters showed clinical significance. All of the clinically significant results can be seen in Tables 1-3.

\section{Discussion}

Ischaemic mitral regurgitation has been described as a disease in which distorted ventricular geometry and impaired function lead to increased systolic tethering and incomplete leaflet closure. A clinical study has shown that the mitral valve area is increased by more than $30 \%$ in patients with inferior $\mathrm{Ml}$ and dilated cardiomyopathy compared with patients with normal hearts at a single evaluation time point [5]. The results of our study demonstrate that patients who have had MI in the inferior wall of the heart are more likely to develop mitral regurgitation of a higher level faster (because of early mitral dilation) and will have lower cardiac output, compared to patients with anterior wall MI. Inferior MI also results in more deaths. Application of an annuloplasty device is an integral component of MV repair as it serves to restore normal shape and size of the MA to optimise MV structure and function. However, there is considerable variation in the intraoperative ring sizing strategy based on MA linear dimensions [2]. Attentive echocardiographic evaluation of a post-MI MA could help cardiologists and cardiosurgeons assess the situation better, resulting in accurate MV ring sizing, quicker action, and fewer deaths. 
Table 2. Parameter difference in four-chamber ultrasound view

\begin{tabular}{lccc}
\hline Variable & $\begin{array}{c}\text { Inferior ST elevation } \\
\text { myocardial infarction }\end{array}$ & $\begin{array}{c}\text { Anterior ST elevation } \\
\text { myocardial infarction }\end{array}$ & P value \\
\hline Diastolic radius of MA & 39.089 & 32.573 & $\mathbf{0 . 0 0 0}$ \\
Systolic radius of MA & 36.223 & 30.935 & $\mathbf{0 . 0 0 0}$ \\
Diastolic area of MA & 12.115 & 8.335 & $\mathbf{0 . 0 0 0}$ \\
Systolic area of MA & 10.146 & 7.577 & $\mathbf{0 . 0 0 1}$ \\
MA contraction & 1.698 & 0.835 & $\mathbf{0 . 0 0 0}$ \\
Distance from AL to anterior leaflet & 28.898 & 30.210 & 0.079 \\
Distance from AL to posterior leaflet & 40.810 & 39.547 & 0.213 \\
Perpendicular of AL to MA & 25.865 & 29.353 & 0.213 \\
\hline
\end{tabular}

All distances are in $\mathrm{mm}$. Area is measured in $\mathrm{mm}^{2}$. Bold numbers show clinical significance; MA — mitral annulus; AL — anterolateral muscle

Table 3. Parameter difference in parasternal ultrasound view

\begin{tabular}{lccc}
\hline Variable & $\begin{array}{c}\text { Inferior ST elevation } \\
\text { myocardial infarction }\end{array}$ & $\begin{array}{c}\text { Anterior ST elevation } \\
\text { myocardial infarction }\end{array}$ & P value \\
\hline Diastolic radius of MA & 36.008 & 33.558 & $\mathbf{0 . 0 0 3}$ \\
Systolic radius of MA & 34.052 & 31.182 & $\mathbf{0 . 0 0 5}$ \\
Diastolic area of MA & 10.136 & 8.876 & $\mathbf{0 . 0 0 3}$ \\
Systolic area of MA & 9.273 & 7.662 & $\mathbf{0 . 0 0 4}$ \\
MA contraction & 1.040 & 1.214 & 0.153 \\
\hline
\end{tabular}

All distances are in $\mathrm{mm}$. Area is measured in $\mathrm{mm}^{2}$. Bold numbers show clinical significance; MA — mitral annulus; $\mathrm{AL}$ - anterolateral muscle

\section{Conclusions}

MA shows some significant changes in both post inferior Ml and post anterior Ml patients. The magnitude of movements of a post inferior MI MA is greatly reduced when compared to MA in patients with anterior MI. While echocardiographically measuring post-MI MA, we decided that inferior $\mathrm{Ml}$ accounts for earlier mitral annular dilatation compared to patients with anterior MI.

Conflict of interest: none declared

\section{References}

1. Nichols M, Townsend N, Scarborough P, Rayner M. Cardiovascular disease in Europe - Epidemiological update. Eur Heart J 2013; 34: 3028-3034.

2. Owais K, Montealegre-Gallegos M, Jeganathan J, Matyal R, Khabbaz KR, Mahmood F. Dynamic changes in the ischemic mitral annulus: Implications for ring sizing. Ann Card Anaesth 2016; 19: 15-19.

3. Silbiger JJ, Bazaz R. Contemporary insights into the functional anatomy of the mitral valve. Am Heart J 2009; 158: 887-895.

4. Pfeffer MA, Braunwald E. Ventricular remodeling after myocardial infarction. Experimental observations and clinical implications. Am Heart J 1990; 81: 1161-1172.

5. Chaput M, Handschumacher MD, Guerrero JL et al. Mitral leaflet adaptation to ventricular remodeling: prospective changes in a model of ischemic mitral regurgitation. Am Heart J 2009; 120: S99-S103. 